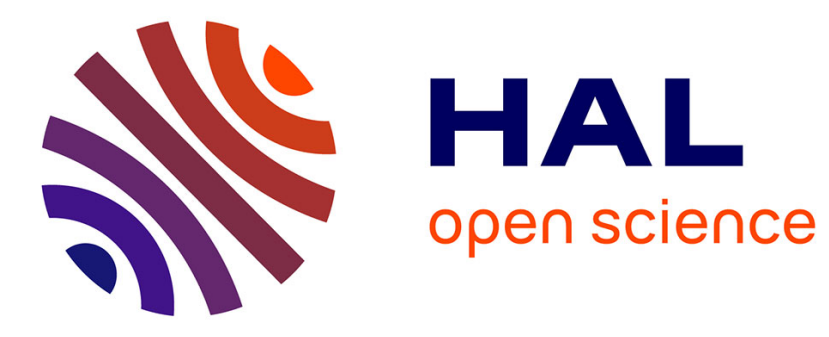

\title{
Rotational Doppler shift upon reflection from a right angle prism
}

Olivier Emile, Janine Emile, Christian Brousseau

\section{To cite this version:}

Olivier Emile, Janine Emile, Christian Brousseau. Rotational Doppler shift upon reflection from a right angle prism. Applied Physics Letters, 2020, 116 (22), 10.1063/5.0009396 . hal-02880694

\section{HAL Id: hal-02880694 https://hal.science/hal-02880694}

Submitted on 1 Jul 2020

HAL is a multi-disciplinary open access archive for the deposit and dissemination of scientific research documents, whether they are published or not. The documents may come from teaching and research institutions in France or abroad, or from public or private research centers.
L'archive ouverte pluridisciplinaire $\mathbf{H A L}$, est destinée au dépôt et à la diffusion de documents scientifiques de niveau recherche, publiés ou non, émanant des établissements d'enseignement et de recherche français ou étrangers, des laboratoires publics ou privés. 


\title{
Rotational Doppler shift upon reflection from a right angle prism
}

\author{
Cite as: Appl. Phys. Lett. 116, 221102 (2020); doi: 10.1063/5.0009396 \\ Submitted: 30 March 2020 - Accepted: 18 May 2020 . \\ Published Online: 2 June 2020

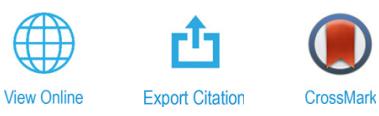

\section{O. Emile, ${ }^{1, a)}$ (D) J. Emile, ${ }^{2}$ and C. Brousseau ${ }^{3}$}

\author{
AFFILIATIONS \\ 'Université de Rennes 1, Campus de Beaulieu, F-35000 Rennes, France \\ ${ }^{2}$ Université de Rennes 1, CNRS IPR UMR 6251, F-35000 Rennes, France \\ ${ }^{3}$ Université de Rennes 1, CNRS IETR UMR 6164, F-35000 Rennes, France
}

a) Author to whom correspondence should be addressed: olivier.emile@univ-rennes7.fr

\begin{abstract}
This Letter reports the observation of a rotational Doppler shift on reflected beams carrying Orbital Angular Momentum (OAM). More precisely, we study the beat frequency of two optical beams carrying OAM with opposite signs, reflected on a right angle prism. We show that the interference of the two beams leads to a daisy-like pattern that rotates at twice the rotating frequency of the prism. The rotational Doppler frequency shift is equal to the OAM topological charge change times the rotational frequency. Possible applications in the positioning and detection of rotation of objects are then discussed.
\end{abstract}

Published under license by AIP Publishing. https://doi.org/10.1063/5.0009396

The picture of the hands of a stopwatch is often proposed to explain the rotational Doppler effect. ${ }^{1,2}$ Indeed, if the stopwatch is placed face up in the center of a turntable, viewed from above, the revolution of the turntable causes an observer in the rest frame to see the hands rotating at a different velocity. This is analogous to the one-day gained by Phileas Fogg on his eastward around-the-world circumnavigation in Jules Verne's famous novel "Around the World in Eighty Days." ${ }^{3}$ In electromagnetism, the rotational Doppler effect manifests itself as a frequency shift. ${ }^{4}$ It can be observed either with the Spin Angular Momentum (SAM) that is related to the polarization of light ${ }^{5,6}$ or with the Orbital Angular Momentum (OAM) of light. ${ }^{7,8}$ It can be detected both in optics and in the radio domain, either in reflection or in transmission, or even when the transmitter and the receiver rotate with respect to each other. ${ }^{9}$ Several applications have been considered, such as probing spinning microparticles, ${ }^{10}$ fluid flow rotation measurements, ${ }^{11,12}$ free-space remote sensing of rotation, ${ }^{13}$ or radar rotational detection, ${ }^{14,15}$ to name a few. However, because the total angular momentum has to change from emission to detection, the rotational Doppler effect has been evidence on scattered light where the OAM changes from $\ell$ to zero ${ }^{16,17}$ or using dedicated rotating object either in transmission ${ }^{7,18}$ or in reflection using phase conjugating mirrors ${ }^{19}$ where the OAM value is reversed. Nevertheless, the rotational Doppler shift upon reflection on simple and widely used components has never been considered. Here, we demonstrate a rotational Doppler effect upon reflection on a right angle prism.
We investigate the frequency shift, depending on the prism angular velocity, for different values of the topological charge of the OAM, before considering possible applications.

The phase of an OAM beam is not uniform on a plane perpendicular to the direction of propagation. It varies from 0 to $2 \ell \pi$ as one makes one complete turn around the direction of propagation. ${ }^{20} \ell$ is called the topological charge of the beam. Experimentally, we will deal with Laguerre-Gaussian (LG) beams that are a special class of OAM beams. The experimental setup is given in Fig. 1. The fundamental mode of a He-Ne laser is converted to a LG mode with a topological charge $\ell$ or a superposition of LG modes thanks to a Spatial Light Modulator (SLM Pluto-2-NIR-011 Holoeye Photonics). This beam impinges then on a right angle prism. It is positioned on a stage that can be rotated at a controlled constant velocity with a motor. The reflected light is analyzed on a screen or using a photodiode connected to a frequency meter (FM).

The physics of the Doppler shift, in general, can be expressed in various equivalent ways. One elegant derivation of the linear Doppler shift in optics is based on the force (radiation pressure force) exerted by the wave on a moving object. ${ }^{21}$ This force equals $P / c$, where $P$ $=N h \nu$ is the power of the incoming beam, $\nu$ is its frequency, $N$ is the number of photons per second, and $c$ is the velocity of light. The power of this force (the rate of doing work) equals $P_{F}=P u / c$, where $u$ is the velocity of the moving object. In order for the power of the force to be conserved, it must correspond to a change in the light 


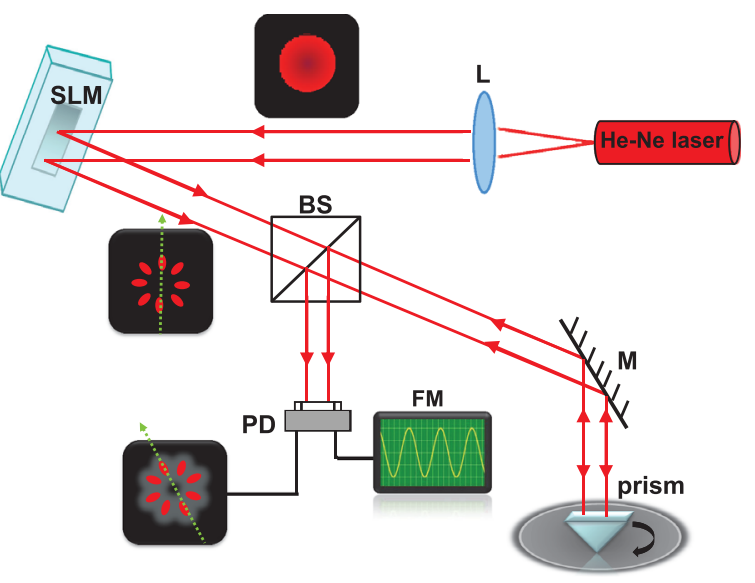

FIG. 1. Experimental setup: the fundamental mode of a He-Ne laser (wavelength $\lambda=633 \mathrm{~nm}$ ) is converted to a superposition of LG beams of opposite charges using a Spatial Light Modulator (SLM). The beam impinges on a rotating right angle prism. The retro reflected light is sent to a screen or to a photodiode connected to a frequency meter (FM). L: lens, BS: beam splitter, M: mirror, and PD: photodiode.

power after interaction with the moving object. Since the number of photons is also conserved, the energy per photon and, thus, the frequency $\nu$ of the wave must change. Equating these to quantities $(P u / c=N h \Delta \nu)$ leads to the usual expression of the linear Doppler shift,

$$
\Delta \nu=\nu u / c .
$$

Besides, an analogous power is defined for a torque applied on a rotating object. Actually, SAM and OAM of light can act as a torque on a macroscopic object. ${ }^{22,23}$ The torque equals $N(s+\ell) \hbar$, where $s= \pm 1$ correspond to the quantum number of spin angular momentum for left- and right-handed circular polarizations, respectively. $\ell$ corresponds to the topological charge of the OAM. The power of the torque is $P_{T}=N(s+\ell) \hbar \Omega$, where $\Omega$ is the angular velocity of the object. Since the number of photons and the power must be conserved, the energy per photon and, thus, the frequency must also change after interaction,

$$
2 \pi \Delta \nu=(s+\ell) \Omega,
$$

which is the usual expression for the rotational Doppler shift. ${ }^{21}$ It is worth noting that the rotational Doppler shift is deeply linked to the torque, as the linear Doppler shift is linked to the force.

Let us now consider the light reflection on a simple object. It could be a mirror, a right angle, a corner cube, or a conical mirror. However, the reflection on a mirror does not change the topological charge nor the polarization of a beam, for a given fixed direction, independent of the direction of the light propagation. There is, thus, no torque associated with the reflection of light on a mirror, and hence, there is no rotational Doppler shift. ${ }^{24,25}$ The same conclusion also holds for a corner cube and for conical reflection. ${ }^{26}$ Nevertheless, the reflection on a right angle prism reverses SAM and OAM.

Actually, the reflection on one side of the prism is equivalent to a reflection on a mirror at $45^{\circ}$ from the beam axis. It does not change the handedness of the beam. Two reflections do not change the handedness either but reverse the propagation direction. Considering a fixed direction, the reflection on a right angle prism changes both SAM and OAM. One may then expect a rotational Doppler shift on a rotating prism. We concentrate on OAM only. This shift should be $2 \Omega$ according to Eq. (2) since, upon reflection, the variation of the OAM is twice the topological charge $(\Delta \ell=2 \ell)$.

Experimentally, we investigate this shift in the beat frequency between two OAM beams with opposite topological charges. ${ }^{27}$ The phase of the electric field of an OAM beam with a topological charge $\ell$ varies as $\ell \theta$, with $\theta$ being the azimuthal angle. ${ }^{28}$ The interference of two beams with opposite topological charges leads to a daisy-like pattern, ${ }^{29,30}$ with the number of petals being equal to twice the topological charge. Upon reflection on a prism rotating at an angular velocity $\Omega$, one of the beam pulsations is shifted by $2 \ell \Omega$, whereas the other one is shifted by $-2 \ell \Omega$. Then, the interference pattern is proportional to

$$
\begin{aligned}
& \left|\mathrm{e}^{-i((\omega+2 \ell \Omega) t+\ell \theta)}+\mathrm{e}^{-i((\omega-2 \ell \Omega) t-\ell \theta)}\right|^{2} \\
& \quad \sim \cos ^{2}(\ell(2 \Omega t+\theta))\left|\mathrm{e}^{-i \omega t}\right|^{2} \sim 1+\cos (2 \ell(2 \Omega t+\theta)),
\end{aligned}
$$

where $\omega=2 \pi \nu$ is the light pulsation and $\ell \theta$ and $-\ell \theta$ account for the phase variation of the OAM beam. The daisy flower rotates at a velocity $2 \Omega$, whatever the value of $\ell$. This is also true for the interference pattern between OAM beams with $\ell$ and $\ell^{\prime}$. In Eq. (3), the term $2 \ell$ is then replaced by $\ell-\ell^{\prime}$.

We first consider the reflection of such a beam on a "static" prism whose position is fixed. However, its position can be adjusted manually. The prism changes the value of the topological charge from $\ell$ to $-\ell$. This reflection, thus, corresponds to a symmetry with respect to the edge of the prism. As the axis of the prism makes an angle $\alpha$ with a symmetry axis of the diffraction pattern of the incident light, the reflected pattern is rotated by an angle $2 \alpha$ (see Fig. 2). To perform this measurement, we take a picture of the interference pattern before and after reflection, and we rotate the two images until they superpose. We then register the value of the rotation angle. This $2 \alpha$ rotation property is valid whatever the topological charge. This is not limited to OAM. The reflection on a right angle prism rotates any image, ${ }^{31}$ even with a dynamical rotation of the prism. However, the connection with the

a)

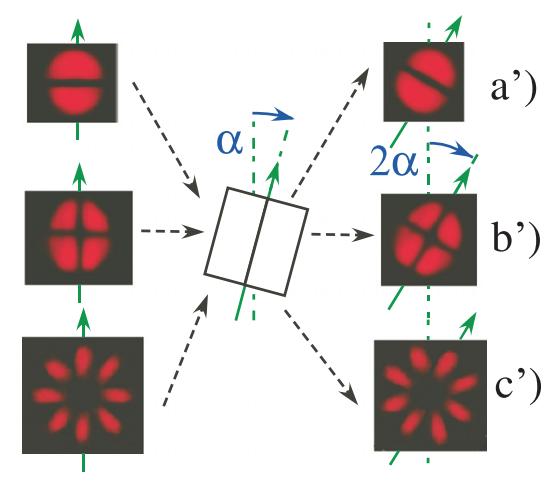

FIG. 2. Reflection of the interference pattern of two OAM beams with opposite charges $\ell$ and $-\ell$, for $\ell=1$ (a, leading to $a^{\prime}$ after reflection), 2 (b, leading to $\left.b^{\prime}\right)$, and 4 (c, leading to $c^{\prime}$ ), on a static right angle prism. As the edge of the prism is rotated by an angle $\alpha$ with respect to a symmetry axis of the interference pattern, the reflected pattern is rotated by $2 \alpha$. 
Doppler effect, which is the main point of this work, has never been established.

As the prism is now rotated at a constant frequency, the interference pattern also rotates. Figure 3 shows pictures of the pattern for the superposition of $\ell=+1$ and $\ell=-1$ OAM beams at different times for a rotation frequency of the prism of $0.15 \mathrm{~Hz}$. The temporal position of the prism for each time is also indicated. Clearly, the interference pattern rotates at a frequency $f_{\text {pat }}$ that is twice the rotation frequency of the prism $f_{\text {prism }}=\Omega / 2 \pi$, as expected from Eq. (3). We have checked that it is also true for higher values of the topological charge. It is worth noting that a linear polarization impinging on the prism would also rotate at $2 \Omega$ and could be detected using a linear polarizer, for example.

We have then continuously varied the rotation frequency of the prism $f_{\text {prism }}$, and we have investigated the rotation frequency of the interference pattern $f_{\text {pat }}$ using the frequency meter, for different values of $\ell$. The variation is perfectly linear and the proportionality coefficient is always exactly equal to 2 , whatever the $\ell$ value, as expected (see Fig. 4).

The topological charge of the two interfering beams can be measured from the number of petals of the daisy flower. From Fig. 4, one knows the rotation frequency of the Daisy flower vs the rotation frequency of the prism. The rotation frequency of the Daisy depends on the respective shifts of the two interfering beams. One can then deduce the variation of the rotational Doppler frequency shift of each beam.

Actually, since the interference pattern rotates at a velocity $2 \Omega$, there is a phase shift $\Omega t$ on the $\ell$ OAM beam and $-\Omega t$ on the $-\ell$ OAM beam that act on the azimuthal angle $\theta$. Because the phase variation associated with the azimuth is $\ell \theta$, one must associate a term $\exp [i \ell(\theta+\Omega t)]$ and term $\exp [-i \ell(\theta+\Omega t)]$ in the expression of the $\ell$ and $-\ell$ OAM electric field, respectively. Finally, one obtains frequency shifts of $\ell \Omega$ for the $\ell$ OAM beam and $-\ell \Omega$ for the $-\ell$ OAM beam. The variation of these shifts vs $f_{\text {prism }}$ is plotted in Fig. 5 . This variation is linear vs the rotation frequency and vs the topological charge, as expected. This shows unambiguously that there is a Doppler shift upon reflection on a right angle prism. This shift $\Delta \nu$ equals twice the rotation frequency of the prism times $\ell, \Delta \nu=2 \ell f_{\text {prism }}$, in agreement with Eq. (2). The rotation of the prism is transposed into the rotation of the interference pattern.

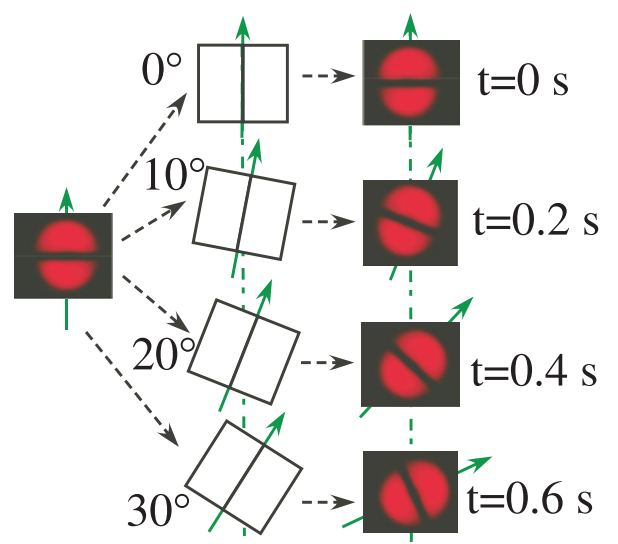

FIG. 3. Image of the reflected interference pattern of two OAM beams, $\ell=1$ and $\ell=-1$, from a rotating right angle prism (rotating frequency $f_{\text {prism }}=0.15 \mathrm{~Hz}$ ), for different times. For each time, the orientation of the prism is also indicated.

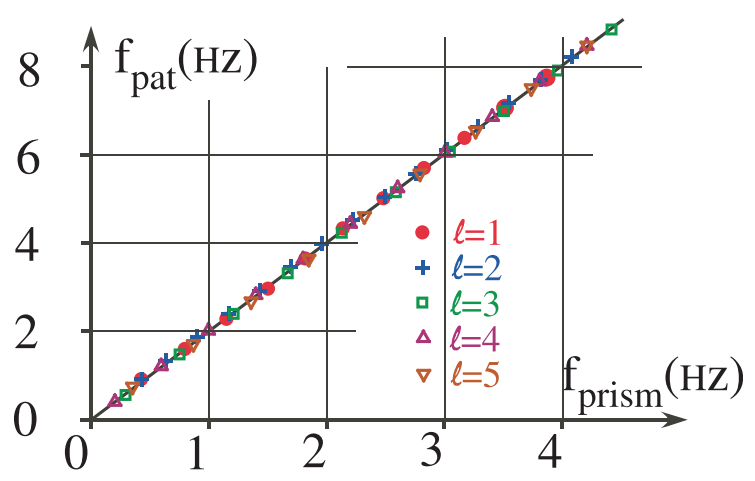

FIG. 4. Rotation frequency $f_{\text {pat }}$ of the interference pattern vs rotation frequency of the prism $f_{\text {prism, }}$, for values of $\ell$ from 1 to 5 .

This rotational Doppler shift on a right angle prism can be used to certainly detect rotation on macroscopic systems and may, thus, lead to various potential applications. Actually, it has been recently argued $^{32}$ that a wide dynamic range, high precision, non-contact, and large bandwidth angular displacement measurement is highly necessary for applications such as industrial control and military equipment. For that purpose, a right angle prism could be installed on the rotation axis of such a system and used to detect its rotation. A servo control could help to position the prism just in the axis of the light source, in order to measure the rotation frequency of the system.

For example, such a prism could be installed on drones or unmanned aerial vehicles that are playing an enhanced role in survey in general and also in telecommunication systems. ${ }^{33}$ Such vehicles face enormous challenges in detecting, identifying, and managing them. Their stability relies on angular displacement sensing. ${ }^{34}$ Since most of the time, feedback systems maintain the drone horizontally, the remaining angle to be determined is the vertical rotation angle. In this case, an OAM laser generating a daisy pattern can be ground based, and a reflecting prism attached to the rear of the drone is shown in Fig. 6(a). Such a system would enable the detection of the rotation angle, either in a static way as it is performed in Fig. 2 or in a dynamical way, measuring the rotational Doppler shift as shown in Fig. 3.

Such a system could also be used in spacecraft rendezvous. Actually, out of the six degrees of freedom between any two systems,

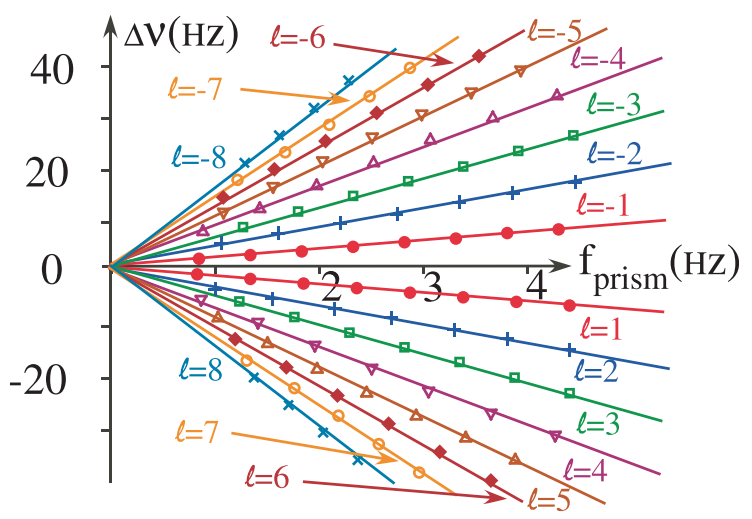

FIG. 5. Rotational Doppler shift of an OAM beam reflected on a prism vs $f_{\text {prism }}$ for values of $\ell$ varying from 1 to 8 . 


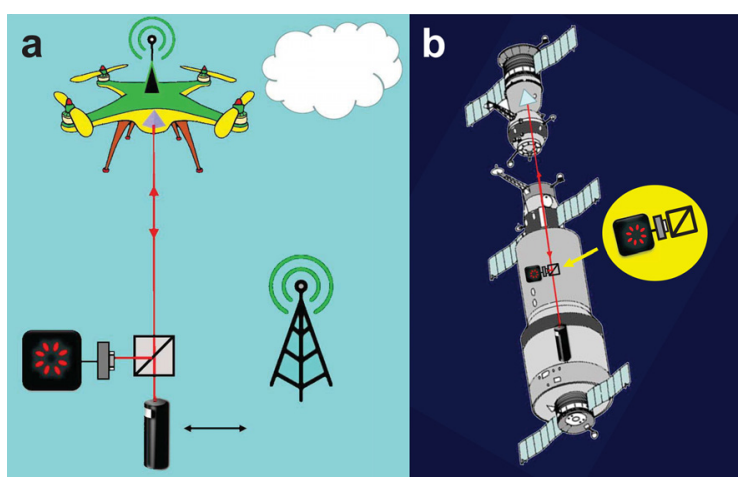

FIG. 6. Example of the application of the rotational Doppler shift in the detection of the rotation of an unmanned aerial vehicle (a) and in the positioning of spacecraft in a space rendezvous (b).

the roll angle is the most difficult to measure. However, in aircraft docking in space rendezvous, the roll angle measurement is essential. ${ }^{35,36}$ OAM daisy pattern reflection on a prism could perform this measurement. For example, the OAM laser system generating the daisy pattern could be attached to one of the spacecraft (let say the chaser), whereas the prism would be linked to the other spacecraft (the target vehicle), as in Fig. 6(b). Then, the rotation and position of the daisy flower would help to exactly position the two spacecraft ready for docking.

To conclude, we have investigated the rotational Doppler shift on a rotating right angle prism. The frequency shift is obtained via the beat frequency between two OAM beams with opposite topological charges. We have shown that the Doppler shift for an OAM beam is equal to the topological charge change times the rotation frequency. However, concerning the interference pattern between these OAM beams, it is a daisy-like flower that rotates at twice the frequency of the rotating prism whatever the topological charge. Thus, when studying rotation through frequency shifts and daisy flower rotations, there is no special need to increase the topological charge to improve the quality of the measurement. Besides, the sensitivity of the rotation measurement is independent of the topological charge. It is only a matter of light intensity as in every interference measurement. ${ }^{29,37}$ Nevertheless, when the Doppler shift is probed using other techniques, such as, for example, directly using a spectrum analyzer in the radio domain, it is worth dealing with higher order modes.

This publication was supported by the European Union through the European Research Development Fund (ERDF) and the French Region of Brittany, Ministry of High Education and Research, Rennes Métropole and Conseil Départemental 35, through The CPER project SOPHIE/STIC and Ondes. We wish to acknowledge valuable discussions with Professor K. Mahdjoubi (Université de Rennes 1).

\section{DATA AVAILABILITY}

The data that support the findings of this study are available from the corresponding author upon reasonable request.

\section{REFERENCES}

'M. Padgett, "Electromagnetism: Like a speeding watch," Nature 443, 924 (2006).
${ }^{2}$ M. Padgett, "A new twist on the Doppler shift," Phys. Today 67(2), 58-59 (2014).

3 J. Verne, Le tour du monde en quatre-vingts jours (Flammarion, 2016).

${ }^{4} J$. Courtial, D. Robertson, K. Dholakia, L. Allen, and M. Padgett, "Rotational frequency shift of a light beam," Phys. Rev. Lett. 81, 4828 (1998).

${ }^{5}$ B. A. Garetz, "Angular Doppler effect," J. Opt. Soc. Am. 71, 609-611 (1981).

${ }^{6} \mathrm{~F}$. Bretenaker and A. L. Floch, "Energy exchanges between a rotating retardation plate and a laser beam," Phys. Rev. Lett. 65, 2316 (1990).

${ }^{7}$ J. Courtial, K. Dholakia, D. Robertson, L. Allen, and M. Padgett, "Measurement of the rotational frequency shift imparted to a rotating light beam possessing orbital angular momentum," Phys. Rev. Lett. 80, 3217-3219 (1998).

${ }^{8}$ L. Fang, M. J. Padgett, and J. Wang, "Sharing a common origin between the rotational and linear Doppler effects," Laser Photonics Rev. 11, 1700183 (2017).

${ }^{9}$ R. Neo, S. Leon-Saval, J. Bland-Hawthorn, and G. Molina-Terriza, "OAM interferometry: The detection of the rotational Doppler shift," Opt. Express 25, 21159-21170 (2017).

${ }^{10}$ D. Phillips, M. Lee, F. Speirits, S. Barnett, S. Simpson, M. Lavery, M. Padgett, and G. Gibson, "Rotational Doppler velocimetry to probe the angular velocity of spinning microparticles," Phys. Rev. A 90, 011801 (2014).

"A. Belmonte, C. Rosales-Guzmán, and J. P. Torres, "Measurement of flow vorticity with helical beams of light," Optica 2, 1002-1005 (2015).

${ }^{12}$ A. Ryabtsev, S. Pouya, A. Safaripour, M. Koochesfahani, and M. Dantus, "Fluid flow vorticity measurement using laser beams with orbital angular momentum," Opt. Express 24, 11762-11767 (2016).

${ }^{13}$ W. Zhang, J. Gao, D. Zhang, Y. He, T. Xu, R. Fickler, and L. Chen, "Free-space remote sensing of rotation at the photon-counting level," Phys. Rev. Appl. 10, 044014 (2018).

${ }^{14} \mathrm{~T}$. Gong, Y. Cheng, X. Li, and D. Chen, "Micromotion detection of moving and spinning object based on rotational Doppler shift," IEEE Microwave Wireless Compon. Lett. 28, 843-845 (2018).

${ }^{15} \mathrm{C}$. Brousseau, K. Mahdjoubi, and O. Emile, "Measurement of the rotational sense and velocity of an object using OAM wave in the radio-frequency band," Electron. Lett. 55, 709-711 (2019).

${ }^{16}$ M. P. Lavery, F. C. Speirits, S. M. Barnett, and M. J. Padgett, "Detection of a spinning object using light's orbital angular momentum," Science 341, 537-540 (2013).

${ }^{17}$ M. P. Lavery, S. M. Barnett, F. C. Speirits, and M. J. Padgett, "Observation of the rotational Doppler shift of a white-light, orbital-angular-momentum-carrying beam backscattered from a rotating body," Optica 1, 1-4 (2014).

${ }^{18}$ B. A. Garetz and S. Arnold, "Variable frequency shifting of circularly polarized laser radiation via a rotating half-wave retardation plate," Opt. Commun. 31, 1-3 (1979).

${ }^{19}$ A. Y. Okulov, "Rotational Doppler shift of a phase-conjugated photon," J. Opt. Soc. Am. B 29, 714-718 (2012).

${ }^{20} \mathrm{~L}$. Allen, M. W. Beijersbergen, R. Spreeuw, and J. Woerdman, "Orbital angular momentum of light and the transformation of Laguerre-Gaussian laser modes," Phys. Rev. A 45, 8185 (1992).

${ }^{21}$ M. Padgett, “Light's twist," Proc. R. Soc. A 470, 20140633 (2014).

${ }^{22}$ G. Delannoy, O. Emile, and A. L. Floch, "Direct observation of a photon spininduced constant acceleration in macroscopic systems," Appl. Phys. Lett. 86, 081109 (2005).

${ }^{23}$ O. Emile, C. Brousseau, J. Emile, R. Niemiec, K. Madhjoubi, and B. Thide, "Electromagnetically induced torque on a large ring in the microwave range," Phys. Rev. Lett. 112, 053902 (2014).

${ }^{24} \mathrm{M}$. Mansuripur, "Angular momentum exchange between light and material media deduced from the Doppler shift," in Optical Trapping and Optical Micromanipulation IX, edited by K. Dholakia and G. C. Spalding (International Society for Optics and Photonics, 2012), Vol. 8458, p. 845805.

${ }^{25} \mathrm{O}$. Emile, C. Brousseau, J. Emile, and K. Mahdjoubi, "Energy and angular momentum transfers from an electromagnetic wave to a copper ring in the UHF band," C. R. Phys. 18, 137-143 (2017).

${ }^{26}$ M. Mansuripur, A. R. Zakharian, and E. M. Wright, "Spin and orbital angular momenta of light reflected from a cone," Phys. Rev. A 84, 033813 (2011).

${ }^{27}$ J. Arlt, M. MacDonald, L. Paterson, W. Sibbett, K. Dholakia, and K. VolkeSepulveda, "Moving interference patterns created using the angular Dopplereffect," Opt. Express 10, 844-852 (2002). 
${ }^{28}$ G. Molina-Terriza, J. P. Torres, and L. Torner, "Twisted photons," Nat. Phys. 3, 305 (2007).

${ }^{29} \mathrm{O}$. Emile and J. Emile, "Naked eye picometer resolution in a Michelson interferometer using conjugated twisted beams," Opt. Lett. 42, 354-357 (2017).

${ }^{30} \mathrm{Q}$. Jia, X. Qiu, Z. Wu, W. Zhang, and L. Chen, "Transferring linear motion of an optical wedge to rotational frequency shift in an orbital angular momentum interferometer," Appl. Phys. Lett. 111, 091102 (2017).

${ }^{31}$ C.-Y. Tsai and P. D. Lin, "Analytical solutions for image orientation changes in prisms," Appl. Opt. 46, 3087-3094 (2007).

${ }^{32}$ L. Chen, D. Zhang, Y. Zhou, C. Liu, and S. Che, "Design of a high-precision and non-contact dynamic angular displacement measurement with dual-laser Doppler vibrometers," Sci. Rep. 8, 9094 (2018).
${ }^{33} \mathrm{~F}$. Xu, T. Hong, J. Zhao, and T. Yang, "Detection and identification technology of rotor unmanned aerial vehicles in 5G scene," Int. J. Distrib. Sens. Networks 15, 1 (2019).

${ }^{34}$ E. Kuantama, T. Vesselenyi, S. Dzitac, and R. Tarca, "PID and fuzzy-PID control model for quadcopter attitude with disturbance parameter," Int. J. Comput. Commun. Control 12, 519-532 (2017).

${ }^{35} \mathrm{~S}$. Li, C. Yang, E. Zhang, and G. Jin, "Compact optical roll-angle sensor with large measurement range and high sensitivity," Opt. Lett. 30, 242-244 (2005).

${ }^{36}$ S. R. Gillmer, X. Yu, C. Wang, and J. D. Ellis, "Robust high-dynamic-range optical roll sensing," Opt. Lett. 40, 2497-2500 (2015).

${ }^{37} \mathrm{P}$. Hariharan, Optical Interferometry, 2nd ed. (Elsevier, 2003). 